\section{Marketing social para doação de sangue: análise da predisposição de novos doadores}

\author{
Social marketing of blood donation: an analysis of \\ new donors' predisposition
}

Marketing social para donar sangre: un análisis de
predisposición de nuevos donantes

\begin{abstract}
This article aims to understand the motivational factors that influence individuals to donate blood, in order to support the social marketing measures for recruiting new donors. Based on theoretical research, a structural model was developed to analyze influences and interactions of constructs in individuals' intentions to donate blood. A field survey used a structured questionnaire with a sample of 346 individuals. The analysis included descriptive analysis, evaluation of psychometric consistency, and structural equation modeling with partial least squares estimation. Fear showed a negative influence and the reference group a positive influence on individual predisposition to donate blood. This indicated that social marketing for blood donation can be more effective in encouraging new donors if it remains focused on these two factors.
\end{abstract}

Blood Donors; Social Marketing; Motivation
Stephanie Ingrid Souza Barboza 1 Francisco José da Costa 1

\section{Resumo}

Este artigo objetiva compreender os fatores motivacionais que influenciam os indivíduos a doarem sangue, com a finalidade de subsidiar as ações de marketing social orientadas à captação de novos doadores. Com base em uma revisão teórica, um modelo de hipóteses foi elaborado, para análise de influências e interações de construtos na formação da predisposição das pessoas a serem doadores de sangue. Foi feito o levantamento de campo por meio de um questionário estruturado, junto a uma amostra de 346 pessoas. Para análise, foram usadas técnicas de análise descritiva, avaliação de consistência psicométrica e modelagem de equações estruturais com estimação por mínimos quadrados parciais. Como resultados, verificou-se principalmente que há uma influência negativa do medo e positiva do grupo de referência na predisposição dos sujeitos em doar sangue. Isso indicou que as ações de marketing social para a doação de sangue são mais eficientes em incentivar novos doadores se mantiverem foco nesses dois fatores.

Doadores de Sangue; Marketing Social; Motivação 


\section{Introdução}

O tema da doação de sangue, recorrente na área de saúde pública, vem despertando interesse como objeto de estudo do marketing social, devido ao entendimento de que os conhecimentos de marketing contribuem para aperfeiçoar as ações de incentivo à doação 1,2 .

No Brasil, a doação de sangue está fundamentada na decisão voluntária dos doadores, o que causa um problema em potencial ${ }^{3}$. Segundo informações do Ministério da Saúde 4, no país são coletadas por ano 3,5 milhões de bolsas de sangue; não obstante, a quantidade ideal de doações é em torno de 5,7 milhões; adicionalmente, embora $1,9 \%$ dos brasileiros seja classificado como doadores, é baixo o nível de frequência de doação, visto que é mais comum a doação apenas uma vez ao ano.

O interesse central deste artigo é analisar os fatores motivacionais que influenciam os indivíduos a adotar comportamentos favoráveis à doação de sangue, no intuito de subsidiar ações mais efetivas pelos agentes públicos e sociais interessados em estimular a doação, considerando a contribuição potencial da teoria de marketing social. Para manter um foco mais específico, a análise está restrita a não doadores e a doadores eventuais. Assim, serão identificados e testados os fatores que influenciam a decisão de possíveis doadores, o que pode contribuir para aperfeiçoar a captação de novos doadores 5 .

Para alcançar esse propósito, foi feito no Referencial Teórico um levantamento acerca da doação de sangue e dos aspectos motivacionais que influenciam a conduta dos potenciais doadores, com a consequente elaboração de um modelo de hipóteses sobre os fatores que influenciam a predisposição dos sujeitos à doação. No item Método são apresentados os procedimentos metodológicos do trabalho de campo, e no item Análise de Dados estão expostos os resultados obtidos e a sua análise. Nas Considerações Finais são tecidas as considerações finais do estudo.

\section{Referencial teórico}

Neste tópico são expostos os aspectos teóricos que compõem o estudo, com ênfase nas questões relativas à doação de sangue, aos fatores motivacionais dos sujeitos e às aplicações de marketing social neste contexto. Ao final, é apresentado o modelo com as hipóteses a serem testadas empiricamente.

\section{O problema da doação de sangue}

O sistema de saúde brasileiro é formado por uma rede complexa de provedores que envolve os segmentos público e privado. Uma das principais funções da saúde pública está na aplicação de métodos de promoção de saúde a fim de proteger, promover e melhorar a saúde e o bem-estar de pessoas afetadas por doenças e outras condições adversas ${ }^{6}$. Nesse contexto, a doação de sangue é um componente essencial para o funcionamento dos sistemas de saúde, sendo de vital importância para a sobrevivência dos indivíduos.

Todavia, o sistema de gerenciamento de sangue ainda é problemático, havendo algumas adversidades que limitam a execução eficiente do processo de captação de sangue, o que sobrecarrega a coordenação e administração das atividades, evidenciando os riscos de vida que os produtos de sangue oferecem, assim como a sua perecibilidade 7 .

Até meados das décadas de 1970 e 1980 no Brasil, a coleta de sangue era realizada por bancos privados que remuneravam os indivíduos, ocorrendo sem regulamentação governamental, o que culminou na constatação de graves problemas neste sistema, obrigando o governo a assumir o exercício da atividade ${ }^{3}$. Já na década de 1990, as organizações públicas desenvolveram programas de recrutamento de doadores entre os familiares e amigos dos indivíduos hospitalizados, os chamados doadores de reposição ${ }^{8}$.

Entende-se que as organizações devem se esforçar para atrair novos doadores, além de reter os doadores regulares, indo além de práticas imediatistas (como a distribuição de materiais educacionais) 9, que suprem apenas as urgências dos momentos de alta demanda, mas não alteram os padrões sociais de comportamento em relação à doação de sangue 10 . Na visão teórica de marketing, isso pode ser alcançado com a aplicação adequada do conhecimento de marketing social a favor da transformação desta problemática.

\section{Marketing social para doação de sangue}

As disfunções e desequilíbrios enfrentados por uma sociedade são motivos de constantes transformações sociais. Para uma orientação mais adequada dessas transformações é desejável um planejamento da mudança, que deve abarcar organizações interessadas em gerir o relacionamento entre o indivíduo e o agente da mudança 10 . Assim, o marketing social se apresenta como tecnologia para mudança de comportamento voluntária, na busca por compreender quais os estímulos que as pessoas precisam para supe- 
rar as barreiras comportamentais que as impedem de aderir a dado comportamento ${ }^{11}$.

Conceitualmente, o marketing social consiste na adaptação das tecnologias do marketing convencional para constituição de programas que objetivam incentivar o comportamento voluntário de um público, com vistas ao bem-estar dos indivíduos adotantes de tal comportamento 11.

Nesse sentido, as ações de marketing social se mostram como um meio adequado para providenciar às políticas públicas alternativas de práticas mais eficientes e voltadas à minimização das dificuldades de captação e retenção dos doadores. A principal contribuição do marketing social para a concretização da mudança social ocorre com a percepção de valor que o indivíduo tem acerca da adoção do comportamento voluntário, o que obriga o agente promotor da mudança a estabelecer com clareza a mudança desejada 12 .

$\mathrm{O}$ marketing social apresenta um modelo de ação que pode elevar a eficiência na promoção da mudança social, ao propor a geração de novos hábitos por meio de uma ideia ofertada 13 . Por exemplo, o altruísmo e o autossacrifício costumam ser vistos como elementos essenciais para que as pessoas adotem determinado comportamento apregoado pelas ações de marketing social 14; deste modo, organismos interessados em uma determinada mudança podem usar as várias ferramentas de marketing existentes para estimular este tipo de comportamento e característica 15 . Sendo esse o caso da doação de sangue, então é evidente o potencial de aplicação de marketing social para tornar os sistemas de promoção da doação mais eficientes 16 .

Pelo que observaram os vários autores da área, a maioria dos programas de marketing social voltados para a saúde pública que explora uma mudança de comportamento para o benefício de terceiros, como é o caso da doação de sangue, gera campanhas que não são direcionadas ao usuário final, já que nem sempre seu comportamento é alterado por meio do marketing social. Portanto, para que o marketing social promova mudanças substanciais no bem-estar social é preciso que suas fronteiras sejam ampliadas, buscando mais do que atingir públicos alvo com mensagens, posto que isto não parece ser suficiente para afastar influências negativas e comportamentos indesejados 15. Isso requer o desenvolvimento de pesquisas associadas aos comportamentos desejados, e esta pesquisa está alinhada a esta demanda.

\section{Fatores motivacionais da doação de sangue}

A contar pelos custos envolvidos na doação voluntária de sangue, é necessário um alto nível de solidariedade e envolvimento psicossocial. Por essa razão, é necessário compreender bem os fatores que influenciam a predisposição das pessoas a serem doadores.

Em geral, o altruísmo emerge como umas das características centrais dos doadores de sangue. O comportamento altruísta é próprio do ato de doar sangue, uma vez que esta ação presume impessoalidade, pois na maioria das vezes se desconhece quem é o doador e o receptor 17,18. Outros motivadores para a doação de sangue são recorrentemente citados, como a consciência da necessidade de sangue dos familiares e amigos, proporcionada pela distribuição eficaz e pelo acesso à informação sobre o assunto 19,20.

Constata-se ainda que o futuro parece exercer influência sobre os doadores de sangue. De um ponto de vista da influência negativa, Shaz et al. 21 citaram como aspectos considerados pelos sujeitos para não doar sangue a falta de aptidão física, o medo do procedimento e de precisar de sangue, a indisponibilidade de tempo, o fato de não ser convidado, e a inconveniência do ato.

Para efeito de visualização conjunta, é adequado investigar a relação das variáveis levantadas com a predisposição dos sujeitos em doar em um só modelo de relações. Nesse intento, foram analisados alguns trabalhos já publicados 1,21,22,23 que integram os diversos fatores que influenciam a doação de sangue, para então delimitar o conjunto de hipóteses proposto, apresentado no modelo estrutural desta pesquisa (Figura 1).

A princípio, Beerli-Palacio \& Martín-Santana 1 indicaram que a informação sobre os procedimentos envolvidos na doação de sangue era o fator que conscientizava o indivíduo a adotar tal comportamento. Esse entendimento constitui a primeira hipótese deste estudo, enunciada a seguir:

H1 - A informação influencia positivamente a predisposição para doar sangue.

Relativo aos fatores intrínsecos que conduzem os sujeitos à doação de sangue, partindo do entendimento de que o desenvolvimento de um comportamento de doador exige do indivíduo uma postura de envolvimento social que pode ser representada por uma conduta altruísta e empática 22,24,25,26,27, e voltada para atender as necessidades dos outros, foi possível definir as três hipóteses a seguir:

H2 - O altruísmo influencia positivamente a predisposição para doar sangue.

H3 - A empatia influencia positivamente a pre-

disposição para doar sangue. 


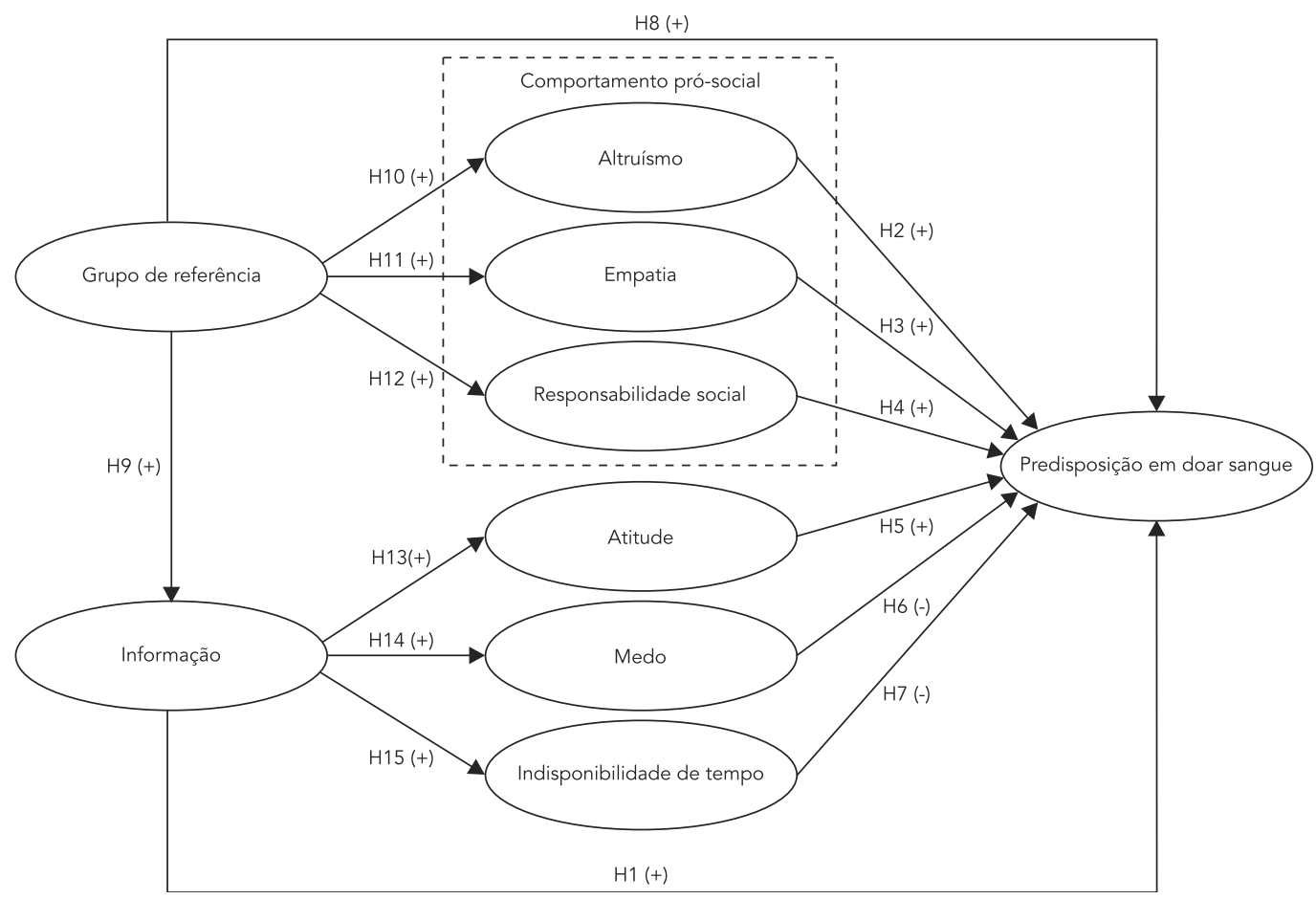

H4 - A responsabilidade social influencia positivamente a predisposição para doar sangue.

De maneira complementar, foi incluído o construto da atitude, na medida em que se apresenta como determinante geral e recorrente da predisposição ${ }^{25}$, ficando definida a hipótese a seguir:

H5 - A atitude das pessoas influencia positivamente a predisposição para doar sangue.

Por se tratar dos inibidores do comportamento de doação de sangue, alguns autores apresentam como construtos o medo, os riscos físicos, e a indisponibilidade de tempo 1,25,28. Aqui também se segue esse entendimento, consolidado nas seguintes hipóteses:

H6 - O medo influencia negativamente o indivíduo a doar sangue.

$\mathrm{H} 7$ - A indisponibilidade de tempo influencia negativamente o indivíduo a doar sangue.

Além da avaliação da própria conduta, os indivíduos consideram os fatores extrínsecos que os influenciam a doar sangue, o que pode ser observado por meio do envolvimento dos sujeitos com as experiências do grupo de referência (família e amigos) 1. Assim, temos enunciada a hipótese a seguir:

H8 - O grupo de referência influencia positivamente a predisposição para doar sangue.

Foi ainda observado na literatura que as experiências do grupo de referência direcionam os indivíduos à doação à medida que, supõe-se, são oferecidas informações sobre a sua própria vivência como doador, ao mesmo tempo em que elucidam alguns procedimentos da doação que favorecem a superação das barreiras dos indivíduos 1 . Desse modo, enunciam-se as hipóteses a seguir: H9 - O grupo de referência influencia positivamente a informação sobre a doação de sangue. $\mathrm{H} 10$ - O grupo de referência influencia positivamente o altruísmo.

H11 - O grupo de referência influencia positivamente a empatia.

$\mathrm{H} 12$ - O grupo de referência influencia positivamente a responsabilidade social.

Adicionalmente, considera-se que a informação sobre a doação de sangue é fator primordial para a adoção do comportamento, e enuncia-se mais uma hipótese: 
H13 - A informação influencia positivamente a atitude do indivíduo de doar sangue.

Por fim, e considerando que há influência do conhecimento sobre os inibidores da doação de sangue 28 , foram definidas mais duas hipóteses: H14 - A informação influencia positivamente o medo do indivíduo.

H15 - A informação influencia positivamente a indisponibilidade de tempo do indivíduo.

A reunião desse conjunto de hipóteses em um só modelo sugere que esses construtos atuam simultaneamente na formação da predisposição à doação. No intuito de testar essas hipóteses, foi então realizado um estudo empírico, sendo apontados no item seguinte os procedimentos metodológicos adotados.

\section{Método}

No trabalho empírico foi usado um método de base predominantemente quantitativo, que é o requerido para testes de modelos como este aqui desenvolvido. Assim, as principais decisões foram concernentes à mensuração, ao design do trabalho de campo e aos procedimentos de análise. A seguir há um detalhamento de cada um desses elementos de decisão.

Quanto à mensuração dos construtos, foram selecionadas escalas previamente utilizadas em outros estudos. Os construtos informação, medo e grupo de referência foram obtidas do estudo de Beerli-Palacio \& Martín-Santana 1. Já as escalas que trataram do altruísmo e responsabilidade social foram extraídas do trabalho de Steele et al. 26, e o construto da empatia foi mensurado com a escala de Falcone et al. 29. O construto da atitude foi obtido em Lemmens et al. 25. Além disso, foi aplicada a escala de Hupfer et al. ${ }^{28}$ para o construto da indisponibilidade de tempo. Para o construto da predisposição à doação foram utilizados itens advindos de vários autores 1,2,9,25.

Como as escalas foram oriundas de estudos em língua inglesa, foi feita a tradução e os itens foram apresentados como afirmações, sendo usada uma escala de verificação de 10 pontos para averiguação de concordância (escala de Likert). A exceção ficou por conta do construto atitude quanto à doação, para o qual foi utilizada uma escala de diferencial semântico de 10 pontos. Com a definição inicial das escalas do instrumento de pesquisa, um questionário foi construído, sendo acrescentadas ainda questões de identificação e descrição da amostra.

Inicialmente, foi decidida a coleta de dados de uma amostra de indivíduos não doadores de sangue e por doadores ocasionais, seguindo o modelo de Fergunson \& Chandler 23. Essa esco- lha é justificada devido à finalidade do estudo de compreender a motivação inicial da adoção do comportamento de doação de sangue (inclusive porque um desafio central do processo de doação é justamente as pessoas se iniciarem como doadores). Para a definição do tamanho da amostra, considerou-se a tradição de pesquisas semelhantes, a demanda da ferramenta estatística, além da capacidade de desenvolvimento de um teste de hipóteses consistente; por estes critérios, a amostra mínima seria de aproximadamente 350 sujeitos (10 por item de mensuração dos construtos que foram operacionalizados conjuntamente ${ }^{28}$ ).

No contato inicial com os potenciais respondentes era inicialmente questionado se estes já haviam doado sangue e quantas vezes. Caso não tivessem doado seriam não doadores, e caso tivessem doado menos de quatro vezes na vida seriam doadores ocasionais (os demais casos seriam doadores regulares e não fariam parte da amostra) 23. Ao final, foram coletados dados de 346 sujeitos. A abordagem ocorreu por acessibilidade, sendo a coleta de dados realizada nas duas maiores cidades da Paraíba (João Pessoa com 27,6\% da amostra e Campina Grande com 29\%, diretamente pelos pesquisadores. Para aumentar a heterogeneidade da amostra, foram ainda disponibilizados questionários pela Internet durante o mês de dezembro de 2011, que correspondeu a $43,4 \%$ dos respondentes, com as respostas estimuladas por pedido enviado por email para aproximadamente mil pessoas. Na avaliação preliminar de potenciais diferenças entre medidas descritivas não foi identificada variação significativa dos dois contextos de coleta, reafirmando a expectativa teórica 30,31 e indicando a possibilidade de operacionalização conjunta dos dados coletados presencialmente e pela Internet.

$\mathrm{Na}$ amostra, observou-se que $53,2 \%$ dos sujeitos realizavam algum tipo de atividade remunerada; $69,7 \%$ tinham renda média familiar superior a R\$ 1.000,00; em termos de faixa etária, a maioria foi de indivíduos mais jovens, principalmente, havendo uma maior participação de pessoas com menos de 35 anos $(81,7 \%)$. Ademais, verificou-se que a maioria foi do sexo feminino $(58,7 \%)$. Esses resultados indicam a heterogeneidade pretendida para a amostra, o que assegura boas condições para os procedimentos de análise empreendidos e minimiza as dificuldades, os potenciais problemas oriundos do procedimento de amostragem por acessibilidade.

Os procedimentos estatísticos que foram empregados para análise dos dados desta pesquisa são os convencionalmente utilizados em pesquisas do gênero, quais sejam: análise da consistência psicométrica dos construtos; análise 
exploratória das variáveis; e, por fim, análise do modelo de hipóteses. Tais procedimentos foram executados nos softwares SPSS (SPSS Inc., Chicago, Estados Unidos) e no SmartPLS (http:/ /www. smartpls.de/forum/, Hamburgo, Alemanha), e com base na literatura especializada neste tipo de análise $32,33,34$.

\section{Análise dos dados}

Neste item, são apresentados os resultados do trabalho de campo. A princípio são expostos os procedimentos de análise exploratória dos construtos, em seguida a análise descritiva das variáveis e, por último, a análise das hipóteses do modelo proposto.

\section{Análise exploratória dos construtos}

Inicialmente, procedeu-se à avaliação da consistência psicométrica dos construtos que compõem o modelo proposto, a partir da obtenção do coeficiente de alpha de Cronbach (desejável acima de 0,60), e a aplicação da análise fatorial exploratória, para análise dos escores fatoriais das variáveis (com valores desejáveis acima de $0,70)$.

De maneira geral, os resultados obtidos apontaram para a consistência em todos os construtos, visto que os valores dos coeficientes do alpha de Cronbach permaneceram acima da margem determinada, sendo o menor valor 0,65 para o construto do altruísmo, indicando para a confiabilidade (de consistência interna) das escalas empregadas.

$\mathrm{Na}$ análise dos escores fatoriais, alguns construtos apresentaram elementos de desajustamento quanto aos escores fatoriais, o que remeteu à necessidade de uma averiguação mais aprofundada dos mesmos. Especificamente no construto empatia, ao ser analisada a extração dos escores fatoriais do conjunto inicial de variáveis, constatou-se que emergiram dois fatores, o que exigiu uma reavaliação do sentido dos enunciados das variáveis de cada fator. Por essa análise, a decisão foi pela separação do construto em duas dimensões, que foram chamados preocupação empática (com quatro variáveis) e compreensão empática (com duas variáveis), o que gerou sub-hipóteses com as mesmas expectativas de influência. Ambos os coeficientes de alpha de Cronbach apresentaram um razoável grau de consistência interna (mínimo de 0,7), o que corroborou a indicação da análise dos escores fatoriais.

Também no construto atitude houve divisão em dois fatores, assim como foi necessária a exclusão de uma variável problemática. Dessa forma, o construto foi operacionalizado por duas dimensões, denominadas de atitude afetiva (duas variáveis) e atitude cognitiva (quatro variáveis). Aqui os resultados foram subdivididos nas hipóteses que tratam da atitude.

A Tabela 1 apresenta as medidas descritivas (média, desvio-padrão, assimetria e curtose) das variáveis finais de cada construto. Pelos resultados da tabela, observa-se que, com relação ao construto da informação, as quatro variáveis apresentaram valores próximos a 7, sendo considerados como médio nível de concordância. No tocante ao desvio-padrão, verificou-se que as variáveis apresentam um valor moderado de dispersão, com escores próximos a 3. Isso mostra que o nível intermediário de conhecimento das pessoas sobre a doação de sangue gera boas expectativas na disseminação deste comportamento.

Quanto aos construtos que compõem o comportamento pró-social, o altruísmo apresentou um alto grau de concordância com as afirmações, com baixa dispersão; o mesmo ocorreu para o construto da responsabilidade social. Para a empatia, as médias das variáveis também se encontraram num patamar de concordância entre elevada e intermediária. Em geral, as medidas são favoráveis à adoção de comportamentos que defendem o bem-estar das outras pessoas.

As médias também se mantiveram num nível intermediário para o construto do grupo de referência (entre 6 e 7). Aqui, os desvios-padrão indicaram uma dispersão mais elevada, sugerindo uma maior variação das respostas dos indivíduos. Para os construtos do medo e da indisponibilidade de tempo, as médias das variáveis foram mantidas num patamar entre 4 e 5 , considerado um baixo nível de concordância com as afirmações. Isso indica que, apesar das barreiras serem percebidas, parte dos respondentes não as considera como os maiores impedimentos para não realizar a doação de sangue. Pelo desvio-padrão foi possível perceber que as respostas apresentam uma dispersão de moderada a elevada.

Concernente às médias do construto da atitude, observou-se que quatro variáveis alcançaram os maiores valores; sugere-se que os respondentes entendem que o ato de doar sangue é uma ação boa, gratificante, vantajosa e estimulante. Já as variáveis que obtiveram médias próximas a 6 demonstram certo receio dos sujeitos ao entenderem a doação de sangue como um ato pouco agradável e confortável. Quanto aos desvios padrão, os resultados mostraram uma dispersão entre baixa e moderada.

Por fim, foi possível observar que as médias das variáveis do construto da predisposição indicaram uma elevada tendência dos sujeitos 
Medidas descritivas das variáveis finais de cada construto.

\begin{tabular}{|c|c|c|c|c|}
\hline Variáveis & Média & DP & Assimetria & Curtose \\
\hline \multicolumn{5}{|l|}{ Informação } \\
\hline Eu sei se posso ou não ser doador de sangue (pois, conheço os requisitos para ser doador de sangue) & 7,24 & 3,01 & $-0,84$ & $-0,55$ \\
\hline Sei bem qual é o destino do sangue que é doado & 7,15 & 2,85 & $-0,65$ & $-0,70$ \\
\hline Sei bem quais as doenças que restringem a doação de sangue & 6,70 & 2,89 & $-0,57$ & $-0,72$ \\
\hline Eu conheço bem os benefícios que receberia se eu fosse doador de sangue regular & 6,72 & 2,98 & $-0,63$ & $-0,80$ \\
\hline \multicolumn{5}{|l|}{ Altruísmo } \\
\hline Eu daria instruções a um estranho (p.ex., atravessar a rua) & 8,68 & 1,89 & $-1,55$ & 1,98 \\
\hline Eu doaria dinheiro para caridade & 8,35 & 1,98 & $-1,14$ & 0,48 \\
\hline Eu doaria algum bem material meu para pessoas carentes & 8,95 & 1,50 & $-1,81$ & 3,90 \\
\hline Eu compraria algum objeto (p.ex., cartões) por saber que é para uma boa causa & 8,44 & 1,81 & $-1,35$ & 1,67 \\
\hline \multicolumn{5}{|l|}{ Empatia } \\
\hline $\begin{array}{l}\text { Quando eu vejo alguém tirar proveito de outra pessoa, sinto-me obrigado(a) a proteger a pessoa } \\
\text { prejudicada }\end{array}$ & 7,90 & 2,24 & $-1,10$ & 0,80 \\
\hline Sinto-me tocado(a) por alguns acontecimentos que presencio & 8,84 & 1,62 & $-1,88$ & 4,16 \\
\hline Quando eu vejo uma pessoa sendo tratada injustamente, eu sinto como se fosse comigo & 8,42 & 1,88 & $-1,23$ & 1,02 \\
\hline Tenho facilidade de entender o ponto de vista de outra pessoa, mesmo quando ela me critica & 7,22 & 2,33 & $-0,73$ & 0,03 \\
\hline Problemas de outras pessoas costumam me comover & 8,31 & 1,95 & $-1,32$ & 1,75 \\
\hline $\begin{array}{l}\text { Antes de pedir a uma pessoa para mudar um comportamento que me incomoda, procuro me colocar no } \\
\text { lugar dela para entender o que a leva ter tal atitude }\end{array}$ & 7,90 & 1,95 & $-1,11$ & 1,41 \\
\hline \multicolumn{5}{|l|}{ Responsabilidade social } \\
\hline Para mim, doar sangue é um dever das pessoas & 7,99 & 2,41 & $-1,40$ & 1,43 \\
\hline Acredito que a doação de sangue é uma ação que salva vidas & 9,62 & 1,13 & $-4,63$ & 26,24 \\
\hline Para mim é preciso se esforçar para ajudar outras pessoas & 8,34 & 2,20 & $-1,53$ & 1,92 \\
\hline Acredito que tenho a responsabilidade e dever de ajudar outras pessoas & 8,50 & 1,92 & $-1,59$ & 2,67 \\
\hline \multicolumn{5}{|l|}{ Grupo de referência } \\
\hline Se eu tivesse um amigo doador de sangue isto me influenciaria a doar & 6,44 & 3,09 & $-0,54$ & $-0,99$ \\
\hline Se eu tivesse um parente doador de sangue isto me influenciaria a doar & 6,93 & 3,08 & $-0,79$ & $-0,69$ \\
\hline \multicolumn{5}{|l|}{ Medo } \\
\hline Pessoalmente, evito ver sangue & 4,06 & 3,24 & 0,67 & $-0,98$ \\
\hline Tenho muito medo de agulhas & 4,28 & 3,25 & 0,55 & $-1,11$ \\
\hline Acho que a doação de sangue me causaria fadiga ou náuseas & 4,31 & 2,87 & 0,48 & $-0,85$ \\
\hline Temo que a doação de sangue me cause alguma dor & 4,08 & 3,09 & 0,66 & $-0,91$ \\
\hline \multicolumn{5}{|l|}{ Indisponibilidade de tempo } \\
\hline Acredito que o tempo de espera para doar sangue é muito longo & 4,66 & 2,85 & 0,23 & $-1,01$ \\
\hline Acho que eu estaria perdendo tempo de estudo ou trabalho se fosse doar sangue & 2,83 & 2,89 & 0,13 & 0,80 \\
\hline Acredito que o processo de doação de sangue pode ser demorado & 4,67 & 2,83 & 0,25 & $-1,02$ \\
\hline \multicolumn{5}{|l|}{ Atitude } \\
\hline Não gratificante/Gratificante & 9,44 & 1,14 & $-2,48$ & 6,11 \\
\hline Ruim/Bom & 9,13 & 1,55 & $-2,60$ & 8,27 \\
\hline Desvantajoso/Vantajoso & 8,70 & 1,67 & $-1,45$ & 2,19 \\
\hline Desagradável/Agradável & 6,98 & 2,37 & $-0,66$ & $-0,10$ \\
\hline Desconfortável/Confortável & 6,18 & 2,55 & $-0,28$ & $-0,79$ \\
\hline Desestimulante/Estimulante & 8,13 & 2,18 & $-1,35$ & 1,38 \\
\hline \multicolumn{5}{|l|}{ Predisposição } \\
\hline Sou uma pessoa predisposta a doar sangue & 8,12 & 2,41 & $-1,35$ & 1,08 \\
\hline Creio que um dia vou tentar doar sangue & 8,75 & 2,26 & $-2,28$ & 4,69 \\
\hline Eu tenho a intenção de doar sangue no futuro & 8,71 & 2,16 & $-2,05$ & 3,90 \\
\hline
\end{tabular}

DP: desvio-padrão. 
pesquisados a adotar um comportamento de doação de sangue, havendo uma dispersão moderada do desvio-padrão.

Pelo que se observou em termos de assimetria e de curtose, foi possível observar que, pelos critérios de extração do SPSS (em que valores de normalidade são entre -1 e +1 ), houve variações entre construtos e entre variáveis nos próprios construtos. Isso sugere que, em geral, não se assegura normalidade das variáveis que deram origem às observações de cada item da amostra. Esse resultado, no entanto, não representa problema em princípio, pois a opção de análise das hipóteses foi pelo método de mínimos quadrados parciais do software SmartPLS. A seguir mostramos os detalhes do procedimento de teste.

\section{Análise das hipóteses do modelo}

Nesta fase, foi inicialmente definido que seriam testados dois modelos: o modelo inicial, com os ajustes oriundos da análise exploratória; e o modelo com novos ajustes e exclusão das hipóteses rejeitadas e das que não influenciam a predisposição. As hipóteses foram testadas valendo-se da técnica de modelagem de equações estruturais, pelo método partial least squares (PLS) do software SmartPLS. A análise central foi feita sobre os valores (re)extraídos dos escores fatoriais (margem de adequação definida: acima de 0,50) e sobre os valores da estatística t de Student, oriundos do procedimento bootstraping realizado pelo mesmo software. Aqui, pelo procedimento estatístico de análise, é assegurado que o valor do coeficiente (beta) de influência é nulo se o valor de t de Student for menor que 1,96 (em módulo), o que garante um nível de significância estatística de $95 \%$ em desfavor da hipótese de que os valores de beta são estatisticamente nulos.

No primeiro modelo, cujos resultados estão expostos na Tabela 2, observou-se que das hipóteses enunciadas foram aceitas seis, com destaque para H6 e H8, que confirmaram a influência do medo e do grupo de referência sobre a predisposição das pessoas a doarem sangue. Considerando ainda as hipóteses aceitas, observa-se que o construto grupo de referência exerce influência na conduta pró-social dos indivíduos, uma vez que os valores de base (valor de beta e valor t) das hipóteses que tratam destas relações (H10, H11b e H12) encontram-se dentro da margem estipulada para não nulidade da relação. Porém, isso não se mostrou suficiente para efeito de geração de predisposição à doação, na medida em que as hipóteses que tratam do altruísmo, da preocupação empática, da compreensão empática e da responsabilidade social exercendo influência na predisposição para a doação de sangue (H2, H3a, H3b e H4) foram rejeitadas. No modelo, o coeficiente de determinação ( $\left.\mathrm{R}^{2}\right)$ do construto predisposição foi de 0,363 .

Todas as demais hipóteses apresentaram indícios de nulidade do efeito previsto. Um destaque aqui vem das hipóteses H5a e H5b, que retrataram as influências da atitude cognitiva e afetiva na predisposição do indivíduo, e que obtiveram valores que não corresponderam à margem definida, o que ressaltou em sua rejeição no estudo. Além disso, nas hipóteses H1, H13a, H13b, H14 e H15, relativas à influência da informação, ficou demonstrado que, mesmo os indivíduos dispondo das informações necessárias para a doação de sangue, não há um considerável efeito desta na mudança comportamental. Naturalmente, esses resultados não eram esperados e, considerando os resultados dos estudos pesquisados na literatura especializada, supõe-se que os resultados tenham associação com a tradição e a cultura nacional brasileira.

Em sequência, o segundo modelo foi testado, envolvendo agora somente as hipóteses aceitas anteriormente e que influenciam a predisposição. Novamente são analisados os valores de influência (beta) e o valor de referência da nulidade estatística ou não desta influência (estatística t). Pelos resultados dessa nova extração, que podem ser observados na Tabela 3, observou-se mais uma vez que as hipóteses $\mathrm{H} 6$ e H8, o medo e o grupo de referência, reafirmam sua influência na predisposição das pessoas a doarem sangue, na medida em que seus valores são significativos para beta, com os valores de t bastante acima da margem de 1,96. Aqui, o coeficiente de determinação $\left(R^{2}\right)$ foi de 0,240 , indicando que, embora haja influência significativa, grande parte da variação da predisposição é explicada por outros fatores.

Portanto, a conclusão foi de que a predisposição do sujeito em adotar um comportamento de doação de sangue é negativamente influenciada pelo medo que o indivíduo alimenta acerca dos procedimentos técnicos aplicados na extração do sangue, assim como dos efeitos colaterais que o ato de doar sangue pode gerar no seu organismo. Em contrapartida, o papel dos familiares e amigos tem influência positiva, o que pode fazer com que o indivíduo supere tais barreiras.

\section{Discussão dos resultados}

Com base nos resultados alcançados, algumas conclusões mais relevantes emergiram. Como destaque inicial, observa-se que, pelas diversas hipóteses rejeitadas, vários dos condicionantes 
Resultados das análises das hipóteses do primeiro modelo (análise fatorial exploratória).

\begin{tabular}{|c|c|c|c|c|}
\hline Hipótese & Representação & $\beta$ & $\begin{array}{c}\text { Valor t do } \\
\text { bootstraping * }\end{array}$ & Situação \\
\hline $\mathrm{H} 1$ & Informação $\longrightarrow$ Predisposição (+) & $-0,001$ & 0,008 & Rejeitada \\
\hline $\mathrm{H} 2$ & Altruísmo $\longrightarrow$ Predisposição (+) & 0,060 & 0,411 & Rejeitada \\
\hline $\mathrm{H} 3 \mathrm{a}$ & Preocupação empática $\longrightarrow$ Predisposição (+) & 0,035 & 0,248 & Rejeitada \\
\hline $\mathrm{H} 3 \mathrm{~b}$ & Compreensão empática $\longrightarrow$ Predisposição (+) & 0,054 & 0,473 & Rejeitada \\
\hline $\mathrm{H} 4$ & Responsabilidade social $\longrightarrow$ Predisposição (+) & 0,197 & 1,198 & Rejeitada \\
\hline $\mathrm{H} 5 \mathrm{a}$ & Atitude cognitiva $\longrightarrow$ Predisposição (+) & 0,202 & 1,339 & Rejeitada \\
\hline $\mathrm{H} 5 \mathrm{~b}$ & Atitude afetiva $\longrightarrow$ Predisposição (+) & 0,058 & 0,423 & Rejeitada \\
\hline H6 & Medo $\longrightarrow$ Predisposição (-) & $-0,263$ & 2,559 & Não rejeitada \\
\hline $\mathrm{H7}$ & Indisponibilidade de tempo $\longrightarrow$ Predisposição (-) & $-0,046$ & 0,445 & Rejeitada \\
\hline $\mathrm{H} 8$ & Grupo de referência $\longrightarrow$ Predisposição (+) & 0,260 & 2,943 & Não rejeitada \\
\hline H9 & Grupo de referência $\longrightarrow$ Informação (+) & 0,095 & 0,904 & Rejeitada \\
\hline $\mathrm{H} 10$ & Grupo de referência $\longrightarrow$ Altruísmo (+) & 0,172 & 1,412 & Rejeitada \\
\hline $\mathrm{H} 11 \mathrm{a}$ & Grupo de referência $\longrightarrow$ Preocupação empática (+) & 0,228 & 1,888 & Rejeitada \\
\hline $\mathrm{H} 11 \mathrm{~b}$ & Grupo de referência $\longrightarrow$ Compreensão empática (+) & 0,266 & 2,742 & Não rejeitada \\
\hline $\mathrm{H} 12$ & Grupo de referência $\longrightarrow$ Responsabilidade social (+) & 0,213 & 2,173 & Não rejeitada \\
\hline $\mathrm{H} 13 \mathrm{a}$ & Informação $\longrightarrow$ Atitude cognitiva (+) & 0,359 & 4,108 & Não rejeitada \\
\hline $\mathrm{H} 13 \mathrm{~b}$ & Informação $\longrightarrow$ Atitude afetiva $(+)$ & 0,409 & 4,408 & Não rejeitada \\
\hline
\end{tabular}

* A extração do SmartPLS não apresenta valores de p, porém a distribuição dos coeficientes extraídos nas 500 reamostragens demandadas é próxima da distribuição t padrão, de modo que o valor de 1,96 é a referência de refutação ou não das hipóteses de nulidade do coeficiente beta.

Tabela 3

Resultados das análises do segundo modelo (final).

\begin{tabular}{lllll}
\hline Hipótese & Representação & $\boldsymbol{\beta}$ & Valor t do bootstraping & Situação \\
\hline H6 & Medo $\longrightarrow$ Predisposição (-) & $-0,341$ & 4,527 & Não rejeitada \\
H8 & Grupo de referência $\rightarrow$ Predisposição $(+)$ & 0,379 & 4,735 & Não rejeitada \\
\hline
\end{tabular}

que são indicados como consistentes em outros estudos não demonstraram impacto na pesquisa empreendida. Pelo esperado, a informação, o altruísmo, a empatia e a responsabilidade social seriam fatores determinantes da motivação à doação, inclusive porque este comportamento está associado à preocupação dos sujeitos em contribuir de maneira efetiva com o bem-estar da sociedade, além desta conduta ser bem vista pelas pessoas que compõem o círculo social dos doadores de sangue.

Possivelmente, a negação da expectativa de influência é explicada por aspectos culturais, o que sugere a necessidade de avaliações em outros estudos. Independentemente, é possível entender que, embora as atividades de educa- ção para doação de sangue desenvolvidas pelas instituições públicas sejam necessárias para que as pessoas tenham conhecimento sobre aspectos de saúde e segurança envolvidos nos procedimentos executados nos hemocentros, para maior eficiência do esforço de captação de doadores há uma necessidade de transformação na abordagem, para que seja enfatizado o que realmente influencia a predisposição à doação.

Alguns dos estudos citados no Referencial Teórico deste artigo indicaram ainda a influência do grupo de referência nos elementos do comportamento pró-social, sendo observado aqui que as referências de familiares e amigos dos sujeitos influenciam parte dos componentes deste comportamento (compreensão empática 
e responsabilidade social); ainda assim, estes comportamentos não exercem influência na predisposição à doação.

Dessa forma, apelos de marketing social baseados em incentivo ao altruísmo e à empatia ou mesmo à responsabilidade social do sujeito não parecem ter efeito algum do ponto de vista de captação de novos doadores. Isso não indica, por outro lado, que esses comportamentos não são relevantes como referência de incentivo à doação, inclusive porque podem ser condicionantes para manutenção de comportamento; é cabido que outros estudos avaliem esta relação neste contexto (de sujeitos já doadores).

Com a confirmação da hipótese H6, que tratou do medo, observou-se que, além das barreiras físicas relacionadas aos procedimentos de doação, existe a questão dos indivíduos não doadores de sangue serem fortemente influenciados pela situação desfavorável do ato de doar sangue, que pode gerar ansiedade, traumas experienciais diversos, assim como a dor e a fadiga oriundas da execução do procedimento em si.

Essa é uma preocupação a ser minimizada, o que pode ser facilitado por ações de marketing social tanto nos indivíduos que já são doadores como nos possíveis novos doadores, haja vista que a segurança e o conforto no desenvolvimento da transfusão de sangue já parecem estar bastante evoluídos, sendo demandado agora um esforço de comunicação que convença a primeira doação, ao mesmo tempo em que providencia uma experiência boa o suficiente para viabilizar a repetição do ato. Nesse sentido, ferramentas de marketing, que são amplamente bem sucedidas no contexto de promoção de consumo, podem facilmente viabilizar um planejamento mais adequado dessa comunicação e da experiência em si.

Por fim, verificou-se ainda que parte da motivação para a predisposição do indivíduo a doar sangue vem da influência de familiares e amigos (hipótese H8). A partir do momento em que os familiares e amigos dos sujeitos já exercem tal comportamento, e os informam acerca dos benefícios gerados pela doação de sangue, há uma superação das barreiras que influenciam negativamente o comportamento de doador. Desse modo, as ações de marketing social podem contribuir na construção de peças de comunicação focadas no incentivo entre amigos e familiares; adicionalmente, o desenvolvimento de ações de integração entre amigos e familiares de doadores em eventos direcionados que podem ser oportunos para captação de novos doadores.

\section{Considerações finais}

Os estudos de doação de sangue e de marketing social convergem na medida em que este último pode contribuir para o planejamento das instituições públicas e para mudanças de comportamento coletivo. Este estudo visou a contribuir nesse contexto, trazendo uma investigação dos fatores que influenciam os indivíduos a doarem sangue, em uma perspectiva típica das análises de marketing.

Foi realizado um levantamento empírico, com a análise dos resultados adotando uma abordagem quantitativa, e os resultados indicaram que dois aspectos são reais influenciadores da predisposição das pessoas a doarem sangue: 0 medo (com influência negativa) e o grupo social (com influência positiva). Esses resultados podem ser levados em conta na análise de contexto e na construção das propostas de ações de organismos públicos orientados à geração de maior captação de doadores.

De maneira geral, este artigo favoreceu um melhor entendimento do contexto geral da doação de sangue, na medida em que analisou e testou alguns aspectos que interferem na tomada de decisão dos não doadores e dos doadores de sangue ocasionais. Nesses termos, o estudo pode servir de referência para outras pesquisas que abordem tal relação. De um ponto de vista prático, os resultados sinalizaram variáveis relevantes para o planejamento de ações de captação de doadores, indicando que os gestores públicos e os profissionais de marketing social devem buscar promover a minimização do medo e influenciar familiares e amigos de não doadores a estimularem as pessoas próximas a se engajarem neste comportamento.

O estudo partiu de uma seleção de não doadores, o que é um aspecto positivo (dada a necessidade de novos doadores), mas isto é também uma limitação, já que os doadores regulares não foram considerados na pesquisa. Torna-se necessário, então, a realização de trabalhos que levem em conta esse grupo (doadores), tendo em vista que sua retenção é outro problema enfrentado pelas instituições de saúde. Adicionalmente, as análises estatísticas não são suficientes para uma compreensão mais plena do comportamento, de modo que é recomendado que estudos qualitativos aprofundem o entendimento do que são os medos, e de quais as melhores estratégias para afastá-los nas ações de captação de doadores. Por fim, pelo fato da amostra ter sido acessada por conveniência, tais resultados não podem ser generalizados; logo, recomendam-se estudos que empreguem procedimentos de amostragem mais rigorosos. 


\section{Resumen}

Este artículo tiene como fin comprender los factores motivacionales que incentivan a las personas a donar sangre, con el fin de subsidiar las acciones de marketing social orientadas a atraer nuevos donantes. A partir de una revisión teórica, se desarrolló un modelo de hipótesis para el análisis de las influencias e interacciones de constructos en la predisposición de las personas para ser donantes de sangre. Se realizó un estudio de campo, usando un cuestionario estructurado con una muestra de 346 sujetos. Para el análisis, se utilizaron técnicas de análisis descriptivo y de consistencia psicométrica, además de la técnica de un modelo de ecuaciones estructurales, con una estimación por mínimos cuadrados parciales. Como resultado, se comprobó principalmente que hay una influencia negativa del miedo y una influencia positiva del grupo, en lo que se refiere a la predisposición de las personas para que donen sangre. Esto indicó que las acciones de marketing social para donar sangre pueden ser más eficaces si incentivan a los nuevos donantes, considerando estos dos factores señalados.

Donantes de Sangre; Mercadeo Social; Motivación

\section{Referências}

1. Beerli-Palacio A, Martín-Santana JD. Model explaining the predisposition to donate blood from the social marketing perspective. International Journal of Nonprofit and Voluntary Sector Marketing 2009; 14:205-14.

2. Reid M, Wood A. An investigation into blood donation intentions among non-donors. International Journal of Nonprofit and Voluntary Sector Marketing 2008; 13:31-43.

3. Gonçalez T, Sabino EC, Chamone DF. Trends in the profile of blood donors at a large blood center in the city of São Paulo, Brazil. Rev Panam Salud Pública 2003; 13:144-8.

4. Departamento de Atenção Especializada, Secretaria de Atenção à Saúde, Ministério da Saúde. Gestão de hemocentros: relatos de práticas desenvolvidas no Brasil. Brasília: Ministério da Saúde; 2010.

5. Ownby HE, Kong F, Watanabe K, Tu Y, Nass CC. Analysis of donor return behavior. Transfusion 1999; 39:1128-35.

6. Atrash HK, Parker CS. The public health response to blood disorders. Am J Prev Med 2010; 38(4 Suppl):S451-5.

7. Li BN, Dong MC, Chao S. On decision making support in blood bank information systems. Expert Syst Appl 2008; 34:1522-32.

\section{Colaboradores}

S. I. S. Barboza colaborou na parte escrita e empírica do artigo. F. J. Costa contribuiu na elaboração da introdução, na orientação dos métodos e das análises e realizou a revisão final.

\section{Agradecimentos}

À Capes pelo apoio financeiro em forma de bolsa concedida à primeira autora; ao CNPq pelo apoio financeiro concedido ao projeto Marketing Social para Doação de Sangue: Uma Análise de Condicionantes Comportamentais dos Doadores, cujo financiamento possibilitou o desenvolvimento desta pesquisa.
8. Carneiro-Proietti AB, Sabino EC, Sampaio D, Proietti FA, Gonçalez TT, Oliveira CDL, et al. Demographic profile of blood donors at three major Brazilian blood centers: results from the international REDS-II study, 2007 to 2008. Transfusion 2010; 50:918-25.

9. France JL, France CR, Himawam LK. Re-donation intentions among experienced blood donors: does gender make a difference? Transfus Apher Sci 2008; 38:159-66.

10. Cunha BGF, Dias MR. Comunicações persuasivas e doação regular de sangue: um estudo experimental. Cad Saúde Pública 2008; 24:1407-18.

11. Andreasen AR. Social marketing in the 21st century. Thousand Oaks: Sage Publications; 2006.

12. Andreasen AR. Social marketing: its definition and domain. Journal of Public Policy \& Marketing 1994; 13:108-14.

13. Smith WA. Social marketing and its potential contribution to a modern synthesis of social change. Soc Mar Q 2002; 8:46-8.

14. Kotler P, Zaltman G. Social marketing: an approach to planned social change. J Mark 1971; 35:3-12.

15. Hastings G, Saren M. The critical contribution of social marketing: theory and application. Marketing Theory 2003; 3:305-22. 
16. Giacomini L, Lunardi Filho WD. Estratégias para fidelização de doadores de sangue voluntários e habituais. Acta Paul Enferm 2010; 23:65-72.

17. Wymer WW. Rethinking the boundaries of social marketing: activism or advertising? J Bus Res 2010; 63:99-103.

18. Fernández-Montoya A. Altruism and payment in blood donation. Transfus Sci 1997; 18:379-86.

19. Suárez IMB, Fernández-Montoya A, Fernández AR, López-Berrio A, Cirrelo-Peñuela M. How regular blood donors explain their behavior. Transfusion 2004; 44:1441-6.

20. Araújo FMR, Feliciano KVO, Mendes MFM, Figueiroa JN. Doadores de sangue de primeira vez e comportamento de retorno no hemocentro público do Recife. Rev Bras Hematol Hemoter 2010; 32:384-90.

21. Shaz BH, Demmons DG, Crittenden CP, Carnevale CV, Lee M, Burnett M, et al. Motivators and barriers to blood donation in African American college students. Transfus Apher Sci 2009; 41:191-7.

22. Sojka BN, Sojka P. The blood donation experience: self-reported motives and obstacles for donating blood. Vox Sang 2008; 94:56-63.

23. Ferguson E, Chandler S. A stage model of blood donor behavior: assessing volunteer behavior. J Health Psychol 2005; 10:359-72.

24. Glynn SA, Kleinman SH, Schreiber GB, Zuck T, McCombs S, Bethel J, et al. Motivations to donate blood: demographic comparisons. Transfusion 2002; 42:216-25.

25. Lemmens KP, Abraham C, Ruiter RAC, Veldhuizen IJT, Dehing CJG, Bos AER, et al. Modelling antecedents of blood donation motivation among non-donors of varying age and education. Br J Psychol 2009; 100:71-90.
26. Steele WR, Schreiber GB, Guiltinan A, Nass C, Glynn SA, Wright DJ, et al. The role of altruistic behavior, empathetic concern, and social responsability motivation in blood donation behavior. Transfusion 2008; 48:43-54.

27. Misje AH, Bosnes V, Gâsdal O, Heier HE. Motivation, recruitment and retention of voluntary nonremunerated blood donors: a survey-based questionnaire study. Vox Sang 2005; 89:236-44.

28. Hupfer ME, Taylor DW, Letwin JA. Understanding Canadian student motivations and beliefs about giving blood. Transfusion 2005; 45:149-61.

29. Falcone EM, Ferreira MC, Luz RCM, Fernandes CS, Faria CA, D'Augustin JF, et al. Inventário de empatia (I.E.): desenvolvimento e validação de uma medida brasileira. Aval Psicol 2008; 7:321-34.

30. Cobanoglu C, Warde B, Moreo PJ. A comparison of mail, fax and web-based survey methods. International Journal of Market Research 2000; 43:441-52.

31. Weigold A, Weigold IK, Russell EJ. Examination of the equivalence of self-report survey-based paperand-pencil and internet data collection methods. Psychol Methods 2013; 18:53-70.

32. Hair JF, Anderson RE, Tatham RL, Black WC. Análise multivariada de dados. 5ạ Ed. Porto Alegre: Bookman; 2005.

33. Hair JF, Hult TM, Ringle CM, Sarstedt M. A Primer on partial least squares structural equation modeling (PLS-SEM). Thousand Oaks: Sage Publications; 2013.

34. Lattin J, Carroll JD, Green PE. Análise de dados multivariados. São Paulo: Cengage Learning; 2011

Recebido em 08/Set/2012

Versão final reapresentada em 05/Set/2013

Aprovado em 08/Jan/2014 